\title{
ON ZERO-DIVISORS IN GROUP RINGS OF GROUPS WITH TORSION
}

\author{
S.V. IVANOV AND ROMAN MIKHAILOV
}

\begin{abstract}
Nontrivial pairs of zero-divisors in group rings are introduced and discussed. A problem on the existence of nontrivial pairs of zero-divisors in group rings of free Burnside groups of odd exponent $n \gg 1$ is solved in the affirmative. Nontrivial pairs of zero-divisors are also found in group rings of free products of groups with torsion.
\end{abstract}

\section{INTRODUCTION}

Let $G$ be a group and $\mathbb{Z}[G]$ denote the group ring of $G$ over the integers. If $h \in G$ is an element of finite order $q>1$ and $X, Y \in \mathbb{Z}[G]$, then we have the following equalities in $\mathbb{Z}[G]$

$$
\begin{aligned}
& X(1-h) \cdot\left(1+h+\cdots+h^{q-1}\right) Y=0, \\
& X\left(1+h+\cdots+h^{q-1}\right) \cdot(1-h) Y=0 .
\end{aligned}
$$

Hence, $X(1-h)$ and $\left(1+h+\cdots+h^{q-1}\right) Y, X\left(1+h+\cdots+h^{q-1}\right)$ and $(1-h) Y$ are left and right zero-divisors of $\mathbb{Z}[G]$ (unless one of them is 0 itself) which we call trivial pairs of zero-divisors associated with an element $h \in G$ of finite order $q>1$. Equivalently, $A, B \in \mathbb{Z}[G]$, with $A B=0, A, B \neq 0$, is a trivial pair of zero-divisors in $\mathbb{Z}[G]$ if there are $X, Y \in \mathbb{Z}[G]$ and $h \in G$ of finite order $q>1$ such that either $A=X(1-h)$ and $B=\left(1+h+\cdots+h^{q-1}\right) Y$ or $A=X\left(1+h+\cdots+h^{q-1}\right)$ and $B=(1-h) Y$.

An element $A \in \mathbb{Z}[G]$ is called a nontrivial left (right) zero-divisor if $A$ is a left (right, resp.) zero-divisor and for every $B \in \mathbb{Z}[G]$ such that $B \neq 0, A B=0$, the pair $A, B$ is not a trivial pair of zero-divisors.

The notorious Kaplansky conjecture on zero-divisors claims that, for any torsionfree group $G$, its integral group ring $\mathbb{Z}[G]$ (or, more generally, its group algebra $\mathbb{F}[G]$ over a field $\mathbb{F}$ ) contains no zero-divisors. In this note, we are concerned with a more modest problem on the existence of zero-divisors in group rings of infinite groups with torsion that would be structured essentially different from the above examples of trivial pairs of zero-divisors. We remark in passing that every pair of zero-divisors in $\mathbb{Z}[G]$ is trivial whenever $G$ is cyclic (or locally cyclic).

Note that if $G$ is a finite group, then every nonzero element $X$ in the augmentation ideal of $\mathbb{Z}[G]$ is a left (right) zero-divisor, because the linear operator $L_{X}: \mathbb{Q}[G] \rightarrow \mathbb{Q}[G]$, given by multiplication $Y \rightarrow X Y(Y \rightarrow Y X$, resp.), has a nontrivial kernel as follows from $\operatorname{dim} L_{X}(\mathbb{Q}[G])<\operatorname{dim} \mathbb{Q}[G]$. Hence, $2-g_{1}-g_{2}$, where $g_{1}, g_{2} \in G$, is a left (right) zero-divisor of $\mathbb{Z}[G]$ unless $g_{1}=g_{2}=1$. On the

2010 Mathematics Subject Classification. Primary 20C07, 20E06, 20F05, 20 F50.

Key words and phrases. Burnside groups, free products of groups, group rings, zero-divisors.

The first named author is supported in part by NSF grant DMS 09-01782. 
other hand, the element $2-g_{1}-g_{2} \in \mathbb{Z}[G]$ is not a trivial left (right) zero-divisor unless $g_{1}, g_{2}$ generate a cyclic subgroup of $G$. Hence, for a finite group $G$, the group ring $\mathbb{Z}[G]$ of $G$ contains no nontrivial zero-divisors if and only if $G$ is cyclic. More generally, if $G$ is a group with a noncyclic finite subgroup $H$, then the element $2-h_{1}-h_{2} \in \mathbb{Z}[G]$, where $h_{1}, h_{2} \in H$, is a nontrivial zero-divisor of $\mathbb{Z}[G]$ unless $h_{1}, h_{2}$ generate a cyclic subgroup of $H$ (for details see the proof of Theorem 2).

However, if $G$ is an infinite torsion (or periodic) group all of whose finite subgroups are cyclic, then the existence of nontrivial pairs of zero-divisors in $\mathbb{Z}[G]$ is not clear. For instance, let $B(m, n)$ be the free Burnside group of rank $m$ and exponent $n$, that is, $B(m, n)$ is the quotient $F_{m} / F_{m}^{n}$ of a free group $F_{m}$ of rank $m$. It is known [13, 8 , that if $m \geq 2$ and $n \gg 1$ is odd, then every noncyclic subgroup of $B(m, n)$ contains a subgroup isomorphic to the free Burnside group $B(\infty, n)$ of countably infinite rank, in particular, every finite subgroup of $B(m, n)$ is cyclic. Note this situation is dramatically different for even $n \gg 1$, see [6].

In this regard and because of other properties of $B(m, n)$, analogous to properties of absolutely free groups, see [13, the first author asked the following question 10. Problem 11.36d]: Suppose $m \geq 2$ and odd $n \gg 1$. Is it true that every pair of zero-divisors in $\mathbb{Z}[B(m, n)]$ is trivial, i.e., if $A B=0$ in $\mathbb{Z}[B(m, n)]$, then $A=X C, B=D Y$, where $X, Y, C, D \in \mathbb{Z}[B(m, n)]$ such that $C D=0$ and the set $\operatorname{supp}(C) \cup \operatorname{supp}(D)$ is contained in a cyclic subgroup of $B(m, n)$ ?

In this paper we will give a negative answer to this question by constructing a nontrivial pair of zero-divisors in $\mathbb{Z}[B(m, n)]$ as follows.

Theorem 1. Let $B(m, n)$ be the free Burnside group of rank $m \geq 2$ and odd exponent $n \gg 1$, and $a_{1}, a_{2}$ be free generators of $B(m, n)$. Denote $c:=a_{1} a_{2} a_{1}^{-1} a_{2}^{-1}$ and let

$$
A:=\left(1+c+\cdots+c^{n-1}\right)\left(1-a_{1} a_{2} a_{1}^{-1}\right), \quad B:=\left(1-a_{1}\right)\left(1+a_{2}+\cdots+a_{2}^{n-1}\right) .
$$

Then $A B=0$ in $\mathbb{Z}[B(m, n)]$ and $A, B$ is a nontrivial pair of zero-divisors in $\mathbb{Z}[B(m, n)]$.

It seems of interest to look at other classes of groups with torsion all of whose finite subgroups are cyclic and ask a similar question on the existence of nontrivial pairs of zero-divisors in their group rings. From this viewpoint, we consider free products of cyclic groups, all of whose finite subgroups are cyclic by the Kurosh subgroup theorem [11, and show the existence of nontrivial pairs of zero-divisors in their group rings. More generally, we will prove the following.

Theorem 2. Let a group $G$ contain a subgroup isomorphic either to a finite noncyclic group or to the free product $C_{q} * C_{r}$, where $C_{n}$ denotes a cyclic group of order $n$ (perhaps, $n=\infty$ ), and $1<\min (q, r)<\infty$. Then the integer group ring $\mathbb{Z}[G]$ of $G$ has a nontrivial pair of zero-divisors.

On the one hand, in view of Theorems 12 one might wonder if there exists a nonlocally cyclic group $G$ with torsion without nontrivial pairs of zero-divisors in $\mathbb{Z}[G]$, in particular, whether there is a free Burnside group $B(m, n)$, where $m, n>1$, with this property. Note that, for every even $n \geq 2$ and $m \geq 2$, the free Burnside group $B(m, n)$ contains a dihedral subgroup, hence, by Theorem $2 \mathbb{Z}[B(m, n)]$ does have a nontrivial pair of zero-divisors.

On the other hand, our construction of nontrivial pairs of zero-divisors in $\mathbb{Z}\left[C_{q} *\right.$ $\left.C_{r}\right]$, where $1<q<\infty, r \in\{2, \infty\}$, and $C_{q}=\langle a\rangle_{q}$ is generated by $a$, produces 
nontrivial pairs of zero-divisors of the form $A B=0$, where $A=(1-a) U, B=$ $U^{-1}\left(\sum_{i=1}^{q} a^{i}\right)$ and $U$ is a unit of $\mathbb{Z}\left[C_{q} * C_{r}\right]$. Thus, our nontrivial pairs of zerodivisors in $\mathbb{Z}\left[C_{q} * C_{r}\right]$ are still rather restrictive and could be named primitive.

Generalizing the definition of a trivial pair of zero-divisors, we say that $A, B \in$ $\mathbb{Z}[G]$, where $A, B \neq 0, A B=0$, is a primitive pair of zero-divisors in $\mathbb{Z}[G]$ if there exists a unit $U$ of $\mathbb{Z}[G]$ such that $A=X U, B=U^{-1} Y$, and $X, Y$ is a trivial pair of zero-divisors in $\mathbb{Z}[G]$. One might conjecture that all pairs of zero-divisors in $\mathbb{Z}[G]$ are primitive whenever $G$ is a free product of cyclic groups. Results and techniques of Cohn [1, 2, see also [3, 4, on units and zero-divisors in free products of rings could be helpful in investigation of this conjecture.

\section{Three Lemmas}

Lemma 1. Suppose that $G$ is a group, $h \in G, H=\langle h\rangle, X \in \mathbb{Z}[G]$, and $C \in \mathbb{Z}[H]$ is not invertible in $\mathbb{Z}[G]$. Then, for every $g \in G$, the left coset $g H$ of $G$ by $H$ is either disjoint from $\operatorname{supp}(X C)$ or $|g H \cap \operatorname{supp}(X C)| \geq 2$.

Proof. Denote $X=\sum_{i=1}^{k} x_{i} C_{i}$, where $C_{i} \in \mathbb{Z}[H]$ and $x_{i} \in G$, so that $x_{i} H \neq x_{j} H$ for $i \neq j$. Then $X C=\sum_{i=1}^{k} x_{i} C_{i} C$ and $\operatorname{supp}(X C)=\bigcup_{i=1}^{k} x_{i} \operatorname{supp}\left(C_{i} C\right)$ is a disjoint union. Since $C$ is not invertible in $\mathbb{Z}[H],\left|\operatorname{supp}\left(C_{i} C\right)\right|>1$ and the result follows.

Recall that a subgroup $K$ of a group $G$ is called antinormal if, for every $g \in G$, the inequality $g K g^{-1} \cap K \neq\{1\}$ implies that $g \in K$.

Lemma 2. Suppose that $G$ is a group, $a, b \in G$, the elements $b, c:=a b a^{-1} b^{-1}$ have order $n>1, d:=a b a^{-1}$, the cyclic subgroups $\langle c\rangle,\left\langle a b^{i}\right\rangle, i=0,1 \ldots, n-1$, are nontrivial, antinormal, and $d \notin\langle c\rangle, c^{j} d \notin\left\langle a b^{i}\right\rangle$ for all $i, j \in\{0,1 \ldots, n-1\}$. Then equalities

$$
\begin{aligned}
& \left(1+c+\cdots+c^{n-1}\right)(1-d)=X C, \\
& (1-a)\left(1+b+\cdots+b^{n-1}\right)=D Y,
\end{aligned}
$$

where $X, Y \in \mathbb{Z}[G], C, D \in \mathbb{Z}[H], H$ is a cyclic subgroup of $G$, and $C D=0$, are impossible.

Proof. Arguing on the contrary, assume that equalities (1)-(2) hold true. Denote $H=\langle h\rangle$. Note that neither $C$ nor $D$ is invertible in $\mathbb{Z}[H]$, because, otherwise, $C D=0$ would imply that one of $C, D$ is 0 which contradicts one of (11)-(2) and the assumptions $a \notin\langle c\rangle, c \neq 1$.

Hence, Lemma 1 applies to the equality (11) and yields that the set

$$
\operatorname{supp}(X C)=\left\{1, c, \ldots, c^{n-1}, d, c d, \ldots, c^{n-1} d\right\}
$$

can be partitioned into subsets of cardinality $>1$ which are contained in distinct left cosets $g H, g \in G$.

Assume that $c^{i_{1}}, c^{i_{2}} \in g H$, where $0 \leq i_{1}<i_{2} \leq n-1$. Then $c^{i_{1}-i_{2}}=h^{k} \neq 1$ and, by antinormality of $\langle c\rangle$, we have $h=c^{i}$ for some $i$. Since $d \in \operatorname{supp}(X C)$, it follows from Lemma 1 that $d h^{j}=d c^{i j} \in \operatorname{supp}(X C)$ with $h^{j} \neq 1$. Hence, either $d c^{i j}=c^{i^{\prime}}$ or $d c^{i j}=c^{i^{\prime}} a$ with $c^{i j} \neq 1$. In either case, we have a contradiction to $d \notin\langle c\rangle$ and antinormality of $\langle c\rangle$.

Now assume that $c^{i_{1}} d, c^{i_{2}} d \in g H$, where $0 \leq i_{1}<i_{2} \leq n-1$. Then $d^{-1} c^{i_{1}-i_{2}} d=$ $h^{k} \neq 1$ and, by antinormality of $\langle c\rangle$, we have $h=d^{-1} c^{i} d$ for some $i$. Since $1 \in$ 
$\operatorname{supp}(X C)$, it follows from Lemma 1 that $h^{j}=d^{-1} c^{i j} d \in \operatorname{supp}(X C)$ with $h^{j} \neq 1$. Hence, either $d^{-1} c^{i j} d=c^{i^{\prime}} \neq 1$ or $d^{-1} c^{i j} d=c^{i^{\prime}} d$. In either case, we have a contradiction to antinormality of $\langle c\rangle$ and $d \notin\langle c\rangle$.

The contradictions obtained above prove that the foregoing partition of the set $\operatorname{supp}(X C)$ consists of two element subsets so that one element belongs to $\left\{1, c, \ldots, c^{n-1}\right\}$ and the other belongs to $\left\{d, c d, \ldots, c^{n-1} d\right\}$. In particular, it follows from $1 \in\left\{1, c, \ldots, c^{n-1}\right\}$ that

$$
h^{k_{1}}=c^{i_{1}} d \neq 1
$$

for some $k_{1}, i_{1}$.

Applying a "right hand" version of Lemma 1, to the equality (2), we analogously obtain that the set

$$
\operatorname{supp}(D Y)=\left\{1, b, \ldots, b^{n-1}, a, a b, \ldots, a b^{n-1}\right\}
$$

can be partitioned into subsets of cardinality $>1$ which are contained in distinct right cosets $H g, g \in G$.

Assume that $b^{i_{1}}, b^{i_{2}} \in H g$, where $0 \leq i_{1}<i_{2} \leq n-1$. Then $b^{i_{1}-i_{2}}=h^{k} \neq 1$ and, by antinormality of $\langle b\rangle$, we have $h=b^{i}$ for some $i$. Since $a \in \operatorname{supp}(D Y)$, it follows from the analog of Lemma 1 (in the "right hand" version) that $h^{j} a=b^{i j} a \in$ $\operatorname{supp}(D Y)$ with $h^{j} \neq 1$. Hence, either $b^{i j} a=b^{i^{\prime}}$ or $b^{i j} a=a b^{i^{\prime}}$. In either case, we have a contradiction to $c \neq 1$ and antinormality of $\langle b\rangle$.

Now assume that $a b^{i_{1}}, a b^{i_{2}} \in H g$, where $0 \leq i_{1}<i_{2} \leq n-1$. Then $a b^{i_{1}-i_{2}} a^{-1}=$ $h^{k} \neq 1$ and, by antinormality of $\langle b\rangle$, we have $h=a b^{i} a^{-1}$ for some $i$. Since $1 \in$ $\operatorname{supp}(D Y)$, it follows from the analog of Lemma 11 that $h^{j}=a b^{i j} a^{-1} \in \operatorname{supp}(D Y)$ with $h^{j} \neq 1$. Hence, either $a b^{i j} a^{-1}=b^{i^{\prime}} \neq 1$ or $a b^{i j} a^{-1}=a b^{i^{\prime}}$. In either case, we have a contradiction to antinormality of $\langle b\rangle$ and $c \neq 1$.

The contradictions obtained above prove that the foregoing partition of the set $\operatorname{supp}(D Y)$ consists of two element subsets so that one element belongs to $\left\{1, b, \ldots, b^{n-1}\right\}$ and the other belongs to $\left\{a, a b, \ldots, a b^{n-1}\right\}$. In particular, it follows from $1 \in\left\{1, b, \ldots, b^{n-1}\right\}$ that

$$
h^{k_{2}}=a b^{i_{2}} \neq 1
$$

for some $k_{2}, i_{2}$.

In view of equalities (3)-(4), we obtain $c^{i_{1}} d a b^{i_{2}}=a b^{i_{2}} c^{i_{1}} d$. Since the subgroup $\left\langle a b^{i_{2}}\right\rangle$ is antinormal, we conclude that $c^{i_{1}} d \in\left\langle a b^{i_{2}}\right\rangle$. This, however, is impossible by assumption and Lemma 2 is proved.

Lemma 3. Suppose $a, b \in G$ are elements of a group $G$ such that the subgroup $\langle a, b\rangle$, generated by $a, b$, is isomorphic to the free product $\langle a\rangle_{q} *\langle b\rangle_{r}$, where $\langle c\rangle_{s}$ denotes a cyclic group of order s generated by $c$ (perhaps, $s=\infty), 1<q<\infty$, $r \in\{2, \infty\}$, and $(q, r)=(2,2)$ if $r=2$. Then the elements

$$
\begin{aligned}
A & :=(1-a)\left(1+(1-a) b\left(\sum_{i=1}^{q} a^{i}\right)\right), \\
B & :=\left(1-(1-a) b\left(\sum_{i=1}^{q} a^{i}\right)\right)\left(\sum_{i=1}^{q} a^{i}\right)
\end{aligned}
$$

satisfy $A B=0$ and form a nontrivial pair of zero-divisors in $\mathbb{Z}[G]$.

Proof. Since

$$
\left(1+(1-a) b\left(\sum_{i=1}^{q} a^{i}\right)\right) \cdot\left(1-(1-a) b\left(\sum_{i=1}^{q} a^{i}\right)\right)=1
$$


it follows that $A B=(1-a)\left(\sum_{i=1}^{q} a^{i}\right)=0$ and $A, B \neq 0$, hence $A, B$ is a pair of zero-divisors in $\mathbb{Z}[G]$. We need to show that $A, B$ is a nontrivial pair of zerodivisors. Arguing on the contrary, assume that $A, B$ is a trivial pair of zero-divisors in $\mathbb{Z}[G]$. Then there is an element $h \in G$ of finite order $s>1$ and $X, Y \in \mathbb{Z}[G]$ such that either

$$
A=X(1-h) \quad \text { and } \quad B=\left(\sum_{i=1}^{s} h^{i}\right) Y
$$

or

$$
A=X\left(\sum_{i=1}^{s} h^{i}\right) \quad \text { and } \quad B=(1-h) Y .
$$

Let $\sigma: \mathbb{Z}[G] \rightarrow \mathbb{Z}$ denote the augmentation homomorphism and $H=\langle h\rangle_{s}$. It follows from definitions (5) - (6) that $\sigma(A)=0$ and $\sigma(B)=q$. On the other hand, it follows that if (7) are true then $\sigma(A)=0$ and if (8) hold then $\sigma(B)=0$. Hence, equalities (7) are true. Looking again at (5)-(6), we see that

$$
\operatorname{supp} A=\left\{1, a, a^{i} b a^{j} \mid i \in\{0,1\}, j \in\{0,1, \ldots, q-1\}\right\} .
$$

By Lemma 1 $\operatorname{supp} A$ can be partitioned into subsets of cardinality $>1$ which are contained in distinct left cosets $g H, g \in G$. Since $1 \in \operatorname{supp} A$, there is also an element $h^{\ell} \neq 1$ in $\operatorname{supp} A$. Now we consider two cases: $r=\infty$ and $(q, r)=(2,2)$.

Suppose $r=\infty$. Since for all $i, j$ elements $a^{i} b a^{j} \in \operatorname{supp} A$ have infinite orders, it follows that $a=h^{\ell}$ for some $\ell$.

Assume $(q, r)=(2,2)$. Then (9) turns into

$$
\operatorname{supp} A=\{1, a, b, a b a, b a, a b\} .
$$

Recall that $\operatorname{supp} A$ can be partitioned into some $k$ subsets $S_{1}, \ldots, S_{k}$ of cardinality $>1$ which are contained in distinct left cosets $g H, g \in G$. Hence, $k \leq 3$. Note if $g_{1}, g_{2} \in\{1, b a, a b\}$ are distinct, then $g_{1}^{-1} g_{2}$ has infinite order in the free product $\langle a\rangle_{2} *\langle b\rangle_{2}$, whence $g_{1}^{-1} g_{2} \notin H$ and $g_{1}, g_{2}$ belong to different sets $S_{1}, \ldots, S_{k}$. Therefore, $k=3$.

Now we can verify that there is only one partition $\operatorname{supp} A=S_{1} \cup S_{2} \cup S_{3}$ such that $S_{1}=\left\{1, g_{2}\right\}, S_{2}=\left\{g_{3}, g_{4}\right\}, S_{3}=\left\{g_{5}, g_{6}\right\}, 1 \in S_{1}, b a \in S_{2}, a b \in S_{3}$, and elements $g_{2}, g_{3}^{-1} g_{4}, g_{5}^{-1} g_{6}$ commute pairwise. This unique partition is the following: $S_{1}=\{1, a\}, S_{2}=\{b, b a\}, S_{3}=\{a b, a b a\}$. Hence, $a=h^{\ell}$.

Thus in either case we have proved that $a=h^{\ell}$ for some $\ell$. Then $\ell q=s$ and

$$
\sum_{i=1}^{s} h^{i}=\left(\sum_{j=0}^{\ell-1} h^{j}\right)\left(\sum_{k=0}^{q-1} h^{\ell k}\right)=\left(\sum_{j=0}^{\ell-1} h^{j}\right)\left(\sum_{k=1}^{q} a^{k}\right) .
$$

Hence,

$$
A\left(\sum_{i=1}^{s} h^{i}\right)=X(1-h)\left(\sum_{i=1}^{s} h^{i}\right)=0 .
$$

On the other hand, it follows from (10) that

$$
\begin{aligned}
A\left(\sum_{i=1}^{s} h^{i}\right) & =A\left(\sum_{k=1}^{q} a^{k}\right)\left(\sum_{j=0}^{\ell-1} h^{j}\right)=(1-a) b\left(\sum_{k=1}^{q} a^{k}\right)^{2}\left(\sum_{j=0}^{\ell-1} h^{j}\right) \\
& =q(1-a) b\left(\sum_{k=1}^{q} a^{k}\right)\left(\sum_{j=0}^{\ell-1} h^{j}\right)=q(1-a) b\left(\sum_{i=1}^{s} h^{i}\right) .
\end{aligned}
$$

Hence, $(1-a) b\left(\sum_{i=1}^{s} h^{i}\right)=0$ in $\mathbb{Z}[G]$ and, for every product $b h^{i}, i=1, \ldots, s$, there is $j$ such that $b h^{i}=a b h^{j}$. This equality implies that $b^{-1} a b=h^{i-j}$, hence $a=h^{\ell}$ commutes with $b^{-1} a b$ in the free product $\langle a\rangle_{q} *\langle b\rangle_{r}$. This is a contradiction which completes the proof. 


\section{Proofs of Theorems}

Proof of Theorem 1. Let $F_{m}=\left\langle b_{1}, b_{2}, \ldots, b_{m}\right\rangle$ be a free group of rank $m$ with free generators $b_{1}, b_{2}, \ldots, b_{m}$ and $B(m, n)=F_{m} / F_{m}^{n}$ be the free $m$-generator Burnside group $B(m, n)$ of exponent $n$, where $F_{m}^{n}$ is the (normal) subgroup generated by all $n$th powers of elements of $F_{m}$. Let $a_{1}, a_{2}, \ldots, a_{m}$ be free generators of $B(m, n)$, where $a_{i}$ is the image of $b_{i}, i=1, \ldots, m$, under the natural homomorphism $F_{m} \rightarrow$ $B(m, n)=F_{m} / F_{m}^{n}$.

Note that if $G=\left\langle g_{1}, g_{2}\right\rangle$ is generated by elements $g_{1}, g_{2}$ and $G$ has exponent $n$, i.e. $G^{n}=\{1\}$, then $G$ is a homomorphic image of $B(m, n)$ if $m \geq 2$. Also, there is a nilpotent group $G_{2, n}=\left\langle g_{1}, g_{2}\right\rangle$ of exponent $n$ and class 2 in which elements $\left[g_{1}, g_{2}\right]:=g_{1} g_{2} g_{1}^{-1} g_{2}^{-1}, g_{2}, g_{1} g_{2}^{i}, i=0, \ldots, n-1$, have order $n$. Therefore, elements $\left[a_{1}, a_{2}\right]:=a_{1} a_{2} a_{1}^{-1} a_{2}^{-1}, a_{2}, a_{1} a_{2}^{i}, i=0, \ldots, n-1$, have order $n$ in $B(m, n)$ if $m \geq 2$. In addition, since $g_{1} g_{2} g_{1}^{-1} \notin\left\langle\left[g_{1}, g_{2}\right]\right\rangle$ and $\left[g_{1}, g_{2}\right]^{j} g_{1} g_{2} g_{1}^{-1} \notin\left\langle g_{1} g_{2}^{i}\right\rangle$ in $G_{2, n}$ for all $i, j \in\{0, \ldots, n-1\}$, it follows that $a_{1} a_{2} a_{1}^{-1} \notin\left\langle\left[a_{1}, a_{2}\right]\right\rangle$ and $\left[a_{1}, a_{2}\right]^{j} a_{1} a_{2} a_{1}^{-1} \notin$ $\left\langle a_{1} a_{2}^{i}\right\rangle$ in $B(m, n)$ for all $i, j \in\{0, \ldots, n-1\}$.

Recall that if $n \gg 1$ is odd (e.g. $n>10^{10}$ as in [12]), then every maximal cyclic subgroup of $B(m, n)$ is antinormal in $B(m, n)$ (this is actually shown in the proof of [13. Theorem 19.4], similar arguments can be found in [5, 9]). Since cyclic subgroups $\left\langle\left[a_{1}, a_{2}\right]\right\rangle,\left\langle a_{1} a_{2}^{i}\right\rangle, i=0, \ldots, n-1$, are of order $n$ and $B(m, n)$ has exponent $n$, it follows that these subgroups $\left\langle\left[a_{1}, a_{2}\right]\right\rangle,\left\langle a_{1} a_{2}^{i}\right\rangle, i=0, \ldots, n-1$, are maximal cyclic and hence are antinormal. Now we can see that all conditions of Lemma 2 are satisfied for elements $a=a_{1}, b=a_{2}, c=\left[a_{1}, a_{2}\right], d=a_{1} a_{2} a_{1}^{-1}$ of $B(m, n)$. Hence, Lemma 2 applies and yields that equalities (1)-(2) are impossible. Furthermore, it is easy to see that $\left(1+c+\cdots+c^{n-1}\right)(1-d) \neq 0$ because $c^{i} d \neq 1, i=0, \ldots, n-1$, and $(1-a)\left(1+b+\cdots+b^{n-1}\right) \neq 0$ because $a b^{j} \neq 1, j=0, \ldots, n-1$.

Finally, we need to show that

$$
\left(1+c+\cdots+c^{n-1}\right)(1-d)(1-a)\left(1+b+\cdots+b^{n-1}\right)=0 .
$$

Note $d=c b$ and $d a=a b$, hence, assuming that $i_{1}, j_{1}, \ldots, i_{4}, j_{4}$ are arbitrary integers that satisfy $0 \leq i_{1}, j_{1}, \ldots, i_{4}, j_{4} \leq n-1$, we have

$$
\begin{aligned}
(1+c & \left.+\cdots+c^{n-1}\right)(1-d)(1-a)\left(1+b+\cdots+b^{n-1}\right)= \\
& =\left(\sum_{i_{1}} c^{i_{1}}-\sum_{i_{2}} c^{i_{2}} d-\sum_{i_{3}} c^{i_{3}} a+\sum_{i_{4}} c^{i_{4}} d a\right)\left(\sum_{j_{1}} b^{j_{1}}\right)= \\
& =\left(\sum_{i_{1}} c^{i_{1}}-\sum_{i_{2}} c^{i_{2}} c b-\sum_{i_{3}} c^{i_{3}} a+\sum_{i_{4}} c^{i_{4}} a b\right)\left(\sum_{j_{1}} b^{j_{1}}\right)= \\
& =\sum_{i_{1}, j_{1}} c^{i_{1}} b^{j_{1}}-\sum_{i_{2}, j_{2}} c^{i_{2}+1} b^{j_{2}+1}-\sum_{i_{3}, j_{3}} c^{i_{3}} a b^{i_{3}}+\sum_{i_{4}, j_{4}} c^{i_{4}} a b^{j_{4}+1}=0 .
\end{aligned}
$$

Thus $\left(1+c+\cdots+c^{n-1}\right)(1-d)$ and $(1-a)\left(1+b+\cdots+b^{n-1}\right)$ is a pair of zero-divisors in $\mathbb{Z}[B(m, n)]$ which is not trivial by Lemma 2 and Theorem 1 is proved.

The idea of the above construction of a nontrivial pair of zero-divisors in $\mathbb{Z}[B(m, n)]$ could be associated with Fox derivatives (which is somewhat analogous to [7, however, no mention of Fox derivatives is made in [7]) and may be described as follows. As above, let $F_{2}=F\left(b_{1}, b_{2}\right)$ be a free group with free generators $b_{1}, b_{2}$. For $w \in F_{2}$, 
consider Fox derivatives $\frac{\partial w}{\partial b_{i}} \in \mathbb{Z}\left[F_{2}\right], i=1,2$. Then

$$
w-1=\frac{\partial w}{\partial b_{1}}\left(b_{1}-1\right)+\frac{\partial w}{\partial b_{2}}\left(b_{2}-1\right)
$$

in $\mathbb{Z}\left[F_{2}\right]$. Letting $w:=\left[b_{1}, b_{2}\right]^{n}$, we observe that $\frac{\partial\left[b_{1}, b_{2}\right]^{n}}{\partial b_{i}}=\left(\sum_{j=0}^{n-1}\left[b_{1}, b_{2}\right]^{j}\right) \frac{\partial\left[b_{1}, b_{2}\right]}{\partial b_{i}}$, $i=1,2$. Hence,

$$
\begin{aligned}
\frac{\partial\left[b_{1}, b_{2}\right]^{n}}{\partial b_{1}} & =\left(\sum_{j=0}^{n-1}\left[b_{1}, b_{2}\right]^{j}\right)\left(1-b_{1} b_{2} b_{1}^{-1}\right), \\
\frac{\partial\left[b_{1}, b_{2}\right]^{n}}{\partial b_{2}} & =\left(\sum_{j=0}^{n-1}\left[b_{1}, b_{2}\right]^{j}\right)\left(b_{1}-b_{1} b_{2} b_{1}^{-1} b_{2}^{-1}\right) .
\end{aligned}
$$

Therefore, taking the image of the equality (11) in $\mathbb{Z}[B(2, n)]$, we obtain

$$
\begin{gathered}
0=\left[a_{1}, a_{2}\right]^{n}-1=\left(\sum_{j=0}^{n-1}\left[a_{1}, a_{2}\right]^{j}\right)\left(1-a_{1} a_{2} a_{1}^{-1}\right)\left(a_{1}-1\right)+ \\
+\left(\sum_{j=0}^{n-1}\left[a_{1}, a_{2}\right]^{j}\right)\left(a_{1}-a_{1} a_{2} a_{1}^{-1} a_{2}^{-1}\right)\left(a_{2}-1\right) .
\end{gathered}
$$

Now multiplication on the right by $\sum_{i=0}^{n-1} a_{2}^{i}$ yields

$$
\left(\sum_{j=0}^{n-1}\left[a_{1}, a_{2}\right]^{j}\right)\left(1-a_{1} a_{2} a_{1}^{-1}\right)\left(a_{1}-1\right)\left(\sum_{i=0}^{n-1} a_{2}^{i}\right)=0
$$

and this is what we have in Theorem 1 .

Analogously, let a group $G=\left\langle a_{1}, a_{2}\right\rangle$ be generated by $a_{1}, a_{2}, a_{2}^{n}=1$ in $G$, a word $w\left(b_{1}, b_{2}\right) \in F\left(b_{1}, b_{2}\right)$ have the property that $w\left(a_{1}, a_{2}\right)=1$ in $G$, and $\theta: \mathbb{Z}\left[F\left(b_{1}, b_{2}\right)\right] \rightarrow \mathbb{Z}[G]$, where $\theta\left(b_{i}\right)=a_{i}, i=1,2$, denote the natural epimorphism. As above, we can obtain

$$
\theta\left(\frac{\partial w\left(b_{1}, b_{2}\right)}{\partial b_{1}}\left(b_{1}-1\right)\left(\sum_{j=0}^{n-1} b_{2}^{j}\right)\right)=\theta\left(\frac{\partial w\left(b_{1}, b_{2}\right)}{\partial b_{1}}\right)\left(a_{1}-1\right)\left(\sum_{j=0}^{n-1} a_{2}^{j}\right)=0 .
$$

This equation can be used for constructing other potentially nontrivial pairs of zerodivisors in $\mathbb{Z}[G]$ (which, however, does not work in case when $G$ is a free product of the form $\left.\left\langle a_{1}\right\rangle *\left\langle a_{2}\right\rangle\right)$.

Proof of Theorem 2. Suppose $G$ is a group and $G$ contains a subgroup $H$ isomorphic either to a finite noncyclic group or to the free product $C_{q} * C_{r}$ of cyclic groups $C_{q}, C_{r}$, where $1<\min (q, r)<\infty$.

First assume $H$ is a finite noncyclic group. Then there are $h_{1}, h_{2} \in H$ such that the subgroup $\left\langle h_{1}, h_{2}\right\rangle$, generated by $h_{1}, h_{2}$, is not cyclic. Since $2-h_{1}-h_{2}$ is a left (right) zero-divisor in $\mathbb{Z}[H], 2-h_{1}-h_{2}$ is also a left (right, resp.) zero-divisor in $\mathbb{Z}[G]$. If $2-h_{1}-h_{2}$ is a trivial left (right, resp.) zero-divisor in $\mathbb{Z}[G]$, then it follows from Lemma 1 that elements $1, h_{1}, h_{2}$ belong to the same coset $g H_{0}\left(H_{0} g\right.$, resp.), where $H_{0}=\left\langle h_{0}\right\rangle$ is cyclic. But then $g \in H_{0}$ and $h_{1}, h_{2} \in H_{0}$, whence the subgroup $\left\langle h_{1}, h_{2}\right\rangle$ is cyclic. This contradiction completes the proof in the case when $H$ is finite noncyclic.

Suppose $C_{q} * C_{r}$ is a subgroup of $G, 1<\min (q, r)<\infty$. We may assume that $q$ is finite. Denote $C_{q}=\langle a\rangle_{q}$ and $C_{r}=\langle b\rangle_{r}$. Note the subgroup $\langle a, b a b a b\rangle$ of $C_{q} * C_{r}$ is isomorphic to the free product $C_{q} * C_{\infty}$ unless $q=r=2$. Therefore, we may assume that $G$ contains a subgroup isomorphic to $C_{q^{\prime}} * C_{r^{\prime}}$, where $q^{\prime}=q>1$ is finite and either $r^{\prime}=\infty$ or $q^{\prime}=r^{\prime}=2$. Now Theorem 2 follows from Lemma 3 ,

\section{REFERENCES}

[1] P. M. Cohn, On the free product of associative rings. II. The case of (skew) fields, Math. Z. 73(1960), 433-456.

[2] P. M. Cohn, On the free product of associative rings. III, J. Algebra 8(1968), 376-383. 
[3] Michael A. Dokuchaev and Maria Lucia Sobral Singer, Units in group rings of free products of prime cyclic groups, Can. J. Math. 50(1998), 312-322.

[4] V. N. Gerasimov, The group of units of a free product of rings, Mat. Sbornik 134(1989), $42-65$.

[5] S. V. Ivanov, Strictly verbal products of groups and a problem of Mal'tcev on operations on groups, Trans. Moscow Math. Soc. 54(1992), 217-249.

[6] S. V. Ivanov, The free Burnside groups of sufficiently large exponents, Internat. J. Algebra Comp. 4(1994), 1-308.

[7] S. V. Ivanov, An asphericity conjecture and Kaplansky problem on zero divisors, J. Algebra 216(1999), 13-19.

[8] S. V. Ivanov, On subgroups of free Burnside groups of large odd exponent, Illinois J. Math. 47(2003), 299-304.

[9] S. V. Ivanov, Embedding free Burnside groups in finitely presented groups, Geom. Dedicata 111(2005), 87-105.

[10] Kourovka Notebook: Unsolved problems in group theory, Novosibirsk, 11th Ed., 1990.

[11] A. G. Kurosh, The theory of groups, Chelsea, 1956.

[12] A. Yu. Ol'shanskii, On the Novikov-Adian theorem, Mat. Sbornik 118(1982), 203-235.

[13] A. Yu. Ol'shanskii, Geometry of defining relations in groups, Nauka, Moscow, 1989; English translation: Math. and Its Applications, Soviet series, vol. 70, Kluwer Acad. Publ., 1991.

Department of Mathematics, University of Illinois, 1409 West Green Street, UrBANA, IL 61801, USA

E-mail address: ivanov@math.uiuc.edu

Steklov Mathematical Institute, Gubkina 8, Moscow, 119991, Russia

E-mail address: romanvm@i.ras.ru 